\title{
DIABETES
}

\section{Regulation of hyperglycaemia-too much can be heart-breaking}

Intensive glucose regulation in patients with hyperglycaemia and acute coronary syndrome (ACS) can be detrimental, a new study published in JAMA Internal Medicine shows.

Patients with ACS are commonly associated with elevated levels of plasma glucose, a recognized determinant of adverse outcomes in patients with and without diabetes mellitus. Nevertheless, whether increased glucose levels contribute to myocardial infarction or are the marker of disease severity in this population of patients is unclear.

\section{4 ...intensive glucose management can be harmful... 77}

In a randomized open-label clinical trial, Dutch researchers investigated the effectiveness and safety of intensive control and regulation of glucose levels in patients with ACS who have hyperglycaemia. Of 1,773 patients with ACS who were admitted to a medical centre between July 2008 and February 2012, 280 patients (218 men, 62 women) with a median age of 65 years were included in the analysis; their glucose levels were $>7.7 \mathrm{mmol} / 1$ - the threshold of impaired glucose toleranceat time of admission for ACS. Patients diagnosed with insulin-dependent diabetes mellitus were excluded from the study.
Half of the participants were randomized to intensive glucose treatment with intravenous insulin for $48 \mathrm{~h}$, and the other 140 participants were randomly allocated to receive conventional glucose management. Glucose levels were targeted to reach normal levels of $4.7-7.7 \mathrm{mmol} / \mathrm{l}$. Patients receiving intensive glucose management had their glucose levels measured hourly until the levels were within the normal range, whereafter the glucose levels were measured every $3 \mathrm{~h}$. Patients treated conventionally had their glucose levels measured at 6, 12, 36 and $72 \mathrm{~h}$ after the onset of ACS symptoms. In this group, insulin treatment was only initiated if glucose levels reached $>16 \mathrm{mmol} / \mathrm{l}$, at which point the patients were transferred to the intensive glucose regulation group.

The study failed to determine whether elevated levels of glucose are a marker of severity of injury or contribute to myocardial infarction. Furthermore, intensive glucose regulation was not associated with a reduced infarct size in hyperglycaemic patients with ACS. Instead, the researchers found that intensive glucose management can be harmful, as the incidence of death or a second spontaneous myocardial infarction was significantly increased in the group that received intensive glucose regulation.

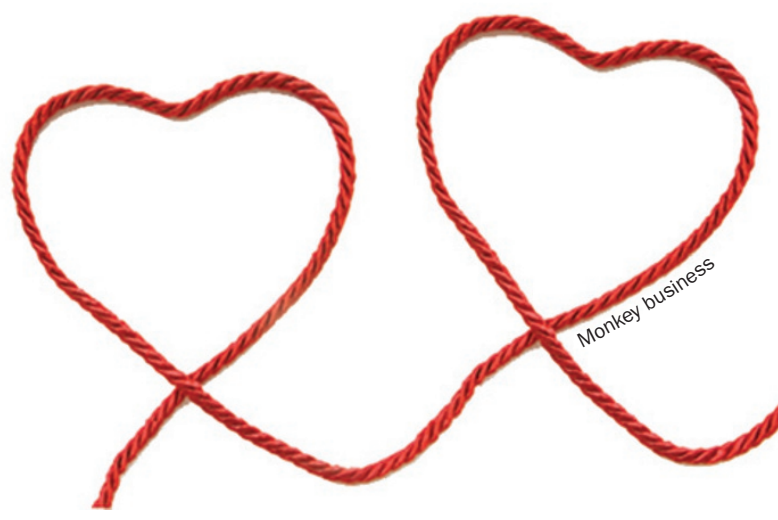

Concluding, the investigators do not recommend intensive insulin therapy in patients with ACS and elevated blood glucose levels; they propose that a "strict, but not too strict, glucose control", as also recommended by the European Society of Cardiology guidelines, be the best practical approach. Further studies are needed to evaluate alternative strategies to obtain optimal glucose levels, especially in patients with ACS who have persistently increased glucose levels after percutaneous coronary intervention.

Elisabeth Kugelberg

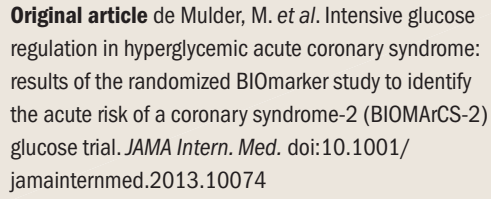

Original article de Mulder, M. et al. Intensive glucose regulation in hyperglycemic acute coronary syndrome: results of the randomized BIOmarker study to identify the acute risk of a coronary syndrome-2 (BIOMArCS-2) glucose trial. JAMA Intern. Med. doi:10.1001/ jamainternmed.2013.10074 